\title{
Game Framework Analysis and Cognitive Learning Theory Providing a Theoretical Foundation for Efficacy in Learning in Educational Gaming
}

\author{
Jason Stratton Davis \\ Durban University of Technology, Durban, South Africa \\ https:/ / orcid.org/0000-0002-3680-2975
}

\begin{abstract}
Several meta-analyses and studies have been undertaken in game-based research, which compare the efficacy of conventional teaching against the introduction of educational games into the classroom. The findings point to educational gaming providing teaching approach that allows for improved efficacy in learning and deeper conceptual understanding. But there is a paucity of research in terms of explaining 'how' and 'why' students learn from games. The mapping out the students' experiences of learning, as a result of an economics gaming intervention, was achieved using research methodology called Interactive Qualitative Analysis (IQA). The findings of the IQA process were then further refined and developed into Game Framework Analysis (GAF) model which points to games providing a learning system that allows for deeper conceptualization of concepts and more meaningful application of knowledge. The question that arose was 'How could this be possible?' Part of the answer is provided by Cognitive Load Theory (CLT) which was developed by Sweller (1988). CLT examines the management of working memory in learning contexts and the resultant effects on learning. Games were found to have in their DNA, the ability to create complex learning environments that can manage the cognitive load in a way that facilitates an optimal usage of working memory, resulting in effective learning.
\end{abstract}

Keywords: Educational Gaming; Cognitive Load Theory; Game Framework Analysis

\section{Introduction}

To date the debate as to whether educational games are an effective medium of instruction that improves student learning continues. But there is increasing evidence from meta-analyzse and systematic reviews on educational gaming that games are beneficial for student learning. But there have been very few studies that have looked at building up a theoretical understanding of why educational games are able to achieve these learning gains which is the focus of this paper. 
This paper starts by looking at literature review of the evidence provided by metaanalyzse, systematic review and research done in the subject of economics. Together, they showed that educational games are an effective pedagogical tool that improves student learning. However, this evidence does not explain why student learning has improved and provides no theoretical underpinning as to reasons for these gains.

It is for the above reasons that we firstly consider a qualitative study that theorizes about students' learning from an Economic gaming intervention. And then secondly, at Cognitive Load Theory (CLT), which gives the theoretical grounding as to why students learn from educational games.

Following this, we then looked briefly at the methodology of the qualitative study which used Interactive Qualitative Analysis (IQA) as its method for collecting data. The IQA process ensures that the participants are actively engaged in the research process and culminates in a visual model of the study. This is then further refined into the development of the Game Framework Analysis (GAF), a model of how and why learning takes place during the play of an educational game.

GAF postulated that a deeper conceptual understanding of the topics taught through the gaming intervention was experienced by the participants in the study. We then began to theorize as to how this was possible which brings us back to CLT. According to CLT, by optimally operating the working memory learning can be improved. This optimization can be enhanced by reducing and managing the intrinsic and extraneous load in the working memory, leaving more space for the germane load (where new knowledge is created).

The paper then discusses at how games can actively reduce the extraneous and intrinsic load in both in their instructional design and deployment. The conclusion reached is that games as an instructional medium have firm theoretical foundations as to why they enhance learning as shown by CLT.

\section{Literature Review}

International literature has over the years shown a growing interest in using games as an instructional medium to improve learning. In this regard, several meta-analyses have been undertaken in game-based research, comparing the efficacy of conventional teaching against that of educational gaming. These metaanalyses that span a broad spectrum of areas of study, ranging from the class to business training. Whether comparing the meta-analyses undertaken on classroom instruction (Randel et al., 1992; Hays, 2005; Vogel et al., 2006; Wouters et al., 2013) or within a business training situation (Sitzmann, 2011) in general, have reached the same conclusion, namely that games are a useful educational tool.

A greater retention of knowledge and higher cognitive gains are the significant benefits arising from the interactive engagement of students when comparing the use of games with conventional teaching (Randel et al.,1992; Hays, 2005; Vogel et al., 2006; Wouters et al., 2013). For utilizing games for business training, Sitzmann (2011) noted that trainees showed higher levels of self-efficacy, declarative knowledge, procedural knowledge, and retention. By introducing educational 
games into the curriculum, Hays (2005) states that one can provide an effective medium of learning for a variety of learners. Furthermore, Wouters et al. (2013), who focused on the cognitive and motivational effects of games, suggest that games could also be used to teach several different subjects. However, the use of games is not a panacea in all situations because they are only effective if they are related to the topic and have clearly defined learning outcomes.

Furthermore, according to Hays (2005), an educational game cannot be a standalone instructional method. Instead, it must be linked directly with the outcomes of the instructional program so that students understand what happens in the game and how it relates to the outcomes through feedback and debriefing. The effectiveness of games is enhanced when used in conjunction with other teaching methods. By embedding games into the curriculum with content that is precisely defined, students not only exhibit a higher level of interest, but their attitude towards the subject improves (Randel et al.,1992; Hays, 2005; Vogel et al., 2006; Wouters et al., 2013).

An exciting aspect that emerged from Sitzmann's (2011) meta-analyses was that the entertainment value of the game was of no importance because the learners gleaned the same amount of information irrespective of whether the game had high or low entertainment value. However, the greater the number of times they repeated the game, the more significant the efficacy. Also, Wouters et al. (2013) noted that learners still gleaned more knowledge with the introduction of educational games as compared to traditional teaching methods. Irrespective of whether they played individually or in groups, by playing the game as a member of a group, they gained more from the learning experience.

A comprehensive systematic review on the effects of games and simulations in higher education by Vlachopoulos and Makri (2017), confirmed the beneficial contribution of game-based learning. In terms of cognitive outcomes, games promoted:

"knowledge acquisition (Smetana \& Bell, 2012; Backlund \& Hendrix, 2013; Clark et al., 2015; Warren et al., 2016), as well as content understanding and concept learning (Connoly et al., 2012; Li E Tsai, 2013; Fu et al., 2016)." (Vlachopoulos \& Makri, 2017, p.8)

According to Vlachopoulos and Makri (2017), there were also significant gains in the affective and behavioral domains. On the affective side, games improved motivation, engagement and satisfaction in learning. While on the behavioral side games provided many opportunities for collaborative learning, increased interactivity and feedback between students, and for the acquisition of social and soft skills (Vlachopoulos \& Makri, 2017).

Moving on from the findings of the meta-analyses and systematic review of educational gaming we now consider specific studies related to the subject within which the study is located, namely Economics. In this field most of most of the research undertaken to-date has been quantitative. It concentrates on comparing the academic performance of students between those who participated in a gaming intervention and those who participated in traditional 'chalk and talk' method. These studies established that the use of games to teach Economics 
improved the student s' achievement (Emerson \& Taylor, 2004; Ball et al., 2006); resulted in better students' retention of course material (Nkonyane \& van Wyk, 2015); stimulated higher student motivation (Gremmen \& van den Brekel, 2013); as well as created a favorable impression of Economics (Tsigaris, 2008; Davis, 2011).

Overall, the meta-analyses and systematic review findings are similar to Economics, that educational gaming provides a teaching approach that allows for improved efficacy in learning and a deeper conceptual understanding. However, there is a lack in the research examining 'how' and 'why' students learn through the playing of educational games. Only then will one be in a better position to gauge the benefits and be able to deploy games so that efficacy of learning is enhanced.

\section{Background}

The study undertaken looked at first year economics students' learning experience of a gaming intervention and forms the basis for this paper. The objectives of this study were to:

- Explore how games influence students' learning, with a view to developing insights for pedagogy; and

- $\quad$ To theorize students' learning from Economics games with the view to deepening existing theory on learning using educational gaming interventions.

The critical questions posed by this research were:

- 'How' do first-year Economics students experience learning in a gaming intervention? and

- 'Why' do students learn from Economics games the way they do?

The study used Interactive Qualitative Analysis (IQA) to map the students' experiences of learning during Economics gaming intervention. Three games were chosen in the field of Micro-Economics, each of which would concentrate on the conceptual understanding of a specific micro-Economics topic, namely:

- Holt (1996) 'Trading in a Pit Market', with the learning outcome to show how markets reach equilibrium through the interaction of buyers (demand) and sellers (supply);

- $\quad$ Kruse et al. (2005) 'Market Forces and Price Ceilings: A Classroom Experiment', with the aim to show the effect of a price ceiling on the market; and

- Neral (1993) 'Widget Production in the Classroom', used to provide students with a tangible experience of the law of diminishing returns.

A first year economics class of 120 participants was then exposed to the Economics gaming intervention. Out of these participants, 24 were randomly invited to participate in the study. However, only 15 of the participants accepted the invitation to participate but this was within recommended the sample size for IQA of 12 to 20 participants (Northcutt \& McCoy, 2004)

During this research process of mapping out the students' experiences into 'how' and 'why' they learnt from Economics gaming intervention, it came to the fore 
that part of reason for the efficacy of learning was underpinned by Sweller's (1998) Cognitive Load Theory (CLT).

\section{Cognitive Load Theory (CLT)}

CLT provides a framework that focuses on the management of working memory as learners interact with the instructional material to achieve effective learning (de Araujo et al., 2016). The working memory is finite, as it can only hold the maximum of seven informational elements at a time (Young et al., 2014). Consequently, if the load on the learner's working memory is exceeded, the result is a decreased efficacy of learning, thereby jeopardizing the student's performance and attainment of knowledge.

CLT, according to Sweller (1988), identifies three cognitive loads which affect the working memory: intrinsic, extraneous and germane. The intrinsic load is both affected by the learner's level of expertise and by the level of difficulty of the task (Sweller et al., 2019). It cannot be affected by instructional interventions. The extraneous load, on the other hand, involves the search for information related to the performance of the task - the cognitive cost of processing information. The extraneous load is affected by how instructions are structured and presented (Hawlitschek \& Joeckel, 2017). Instructional techniques can, therefore, increase the extraneous load:

"by providing insufficient guidance, thereby forcing learners to employ weak problem-solving methods such as trial and error, or to search for the information necessary to complete the task." (Young et al., 2014, p. 5).

The third cognitive load, the germane load, is the working memory resources taken up by processing new information (Ouellette et al., 2019). This involves the learner's mental efforts to construct new schemata (knowledge). As a result, there is a need to have more of the working memory dedicated to the germane load to facilitate deeper conceptual understanding.

The only way to achieve this is by managing both the extraneous and intrinsic loads so that the germane load takes up the remaining space left by the sum of the intrinsic and extraneous loads.

The initial focus of the research was solely on minimizing the extraneous load as the intrinsic load is assumed to be fixed, thereby making more working memory available for the expansion of the germane load. However, later research points to the fact that the intrinsic load may not be fixed and can be manipulated in a manner that creates further room in the working memory for the germane load to operate in. Therefore, one should look at managing both the intrinsic and extraneous loads in a manner that allows for more working memory to be devoted to the germane load.

\section{Managing the extraneous load}

The design of instructional material should, according to CLT, aim to reduce the extraneous load by managing the intrinsic load and optimizing the germane load (de Araujo et al., 2016). Table 1 below illustrates possible ways in which to reduce the extraneous load. 
Table I. Some Effects Studied by Cognitive Load Theory and Why They Reduce Extraneous Loads (van Merrienboer \& Sweller ,2005, p. 151.)

\begin{tabular}{|c|c|c|}
\hline Effect & Description & Extraneous load \\
\hline Goal-free effect & $\begin{array}{l}\text { Replace } \\
\text { problems with } \text { gonventional } \\
\text { problems that } \\
\text { learners with an a-specific } \\
\text { goal }\end{array}$ & $\begin{array}{l}\text { Reduces } \\
\text { cogtraneous } \\
\text { relating a current problem } \\
\text { state to a goal state and } \\
\text { attempting to reduce } \\
\text { differences between them; } \\
\text { focuses learner's attention on } \\
\text { problem states and available } \\
\text { operators }\end{array}$ \\
\hline $\begin{array}{l}\text { Worked example } \\
\text { Effect }\end{array}$ & $\begin{array}{l}\text { Replace } \\
\text { problems with wonked } \\
\text { examples that must be } \\
\text { carefully studied }\end{array}$ & $\begin{array}{l}\text { Reduces extraneous } \\
\text { cognitive load caused by } \\
\text { weak-method problem- } \\
\text { solving; focuses learner's } \\
\text { attention on problem states } \\
\text { and useful solution steps }\end{array}$ \\
\hline $\begin{array}{l}\text { Completion } \\
\text { problem effect }\end{array}$ & $\begin{array}{l}\text { Replace conventional } \\
\text { problems with completion } \\
\text { problems, providing a partial } \\
\text { solution that must be } \\
\text { completed by the learners }\end{array}$ & $\begin{array}{l}\text { Reduces extraneous } \\
\text { cognitive load because giving } \\
\text { part of the solution reduces } \\
\text { the size of the problem space; } \\
\text { focus attention on problem } \\
\text { states and useful solution } \\
\text { steps }\end{array}$ \\
\hline $\begin{array}{l}\text { Split attention } \\
\text { Effect }\end{array}$ & $\begin{array}{l}\text { Replace multiple sources of } \\
\text { information (frequently } \\
\text { pictures and accompanying } \\
\text { text) with a single, integrated } \\
\text { source of information }\end{array}$ & $\begin{array}{l}\text { Reduces } \\
\text { cognitive load because there } \\
\text { is no need to mentally } \\
\text { integrate the information } \\
\text { sources }\end{array}$ \\
\hline Modality effect & $\begin{array}{l}\text { Replace a written } \\
\text { explanatory text and another } \\
\text { source of visual information } \\
\text { such as a diagram (unimodal) } \\
\text { with a spoken explanatory } \\
\text { text and a visual source of } \\
\text { information (multimodal) }\end{array}$ & $\begin{array}{l}\text { Reduces extraneous } \\
\text { cognitive load because the } \\
\text { multimodal presentation } \\
\text { uses both the visual and } \\
\text { auditory processor of } \\
\text { working memory }\end{array}$ \\
\hline $\begin{array}{l}\text { Redundancy } \\
\text { Effect }\end{array}$ & $\begin{array}{l}\text { Replace multiple sources of } \\
\text { information that is self- } \\
\text { contained (i.e., they can be } \\
\text { understood on their own) } \\
\text { with one source of } \\
\text { information }\end{array}$ & 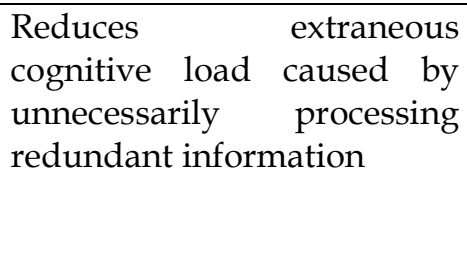 \\
\hline
\end{tabular}

\section{Managing the intrinsic load}

The intrinsic load in the working memory can be reduced through the following interventions, namely by increasing the student's expertise and reducing the difficulty of the task. Both of these interventions have to be considered in the development and design of the instructional material. Hence the positioning of instructional material after the requisite skills and knowledge have been acquired to complete the task would reduce the intrinsic load. 
Furthermore, by reducing the difficulty of the task through minimizing the interactivity amongst the elements - the more interactivity between the elements, the more complex the task, and the higher the level of expertise required to complete the task - the higher the intrinsic load. However, if the task is then deconstructed into discrete elements that can be processed serially, it would thereby minimize the intrinsic load. Then, at a later stage, the materials are presented in the full complexity (Klepsch \& Seufert, 2020).

\section{Methodology}

The study used Interactive Qualitative Analysis (IQA) as the means of collecting, analyzing and interpreting the data. IQA falls within the social constructivist paradigm where participants analyze and interpret the data research. The first phase of the IQA process utilizes focus groups in which the participants (constituents):

"themselves perform the first steps of analysis by organizing their discourse into categories of meaning called affinities" (Northcutt \& McCoy, 2004, p. 44).

The causal relationships between the affinities are then mapped out by the participants, from which a conceptual map is derived. This is referred to as an Inter-Relationship Diagram (IDR) which is then developed into a Systems Influence Diagram (SID) - the final product of IQA. The SID is a visual representation of the drivers and the outcomes: the influences between the affinities, causes and effects.

The second phase of IQA involves individual semi-structured interviews where, the affinities are further explored on a one-on-one basis. This not only allows for personal perspectives, but also encourages a greater level of detail, richness and depth of description of the data.

Throughout the IQA research process, participants themselves are responsible for generating the affinities and analyzing their causal relationships. This differs significantly from traditional qualitative research, where the researcher generates the data. Concerning IQA, the researcher's role changes as they "move(s) from designer to facilitator, teaching the group members the process and guiding them to generate and analyze their own data with minimal external influence" (Northcutt \& McCoy, 2004, p. 44).

In this way, the opportunities for researcher bias is minimized.

\section{Presentation of outcome of study - GAF}

The outcome of the research was the development of the Game Framework Analysis (GAF). GAF provides a framework with which one can analyze 'how' and 'why' students learn from a gaming intervention. 


\section{Tour Trough GAF}

\begin{tabular}{c} 
GAME SPACE - EDUCATIONAL GAMING \\
ENVIRONMENT \\
PRIMARY DRIVER: USE MORE GAMES \\
PLUS \\
PRIMARY OUTCOME: DID NOT FEEL LIKE A LESSON \\
\hline
\end{tabular}

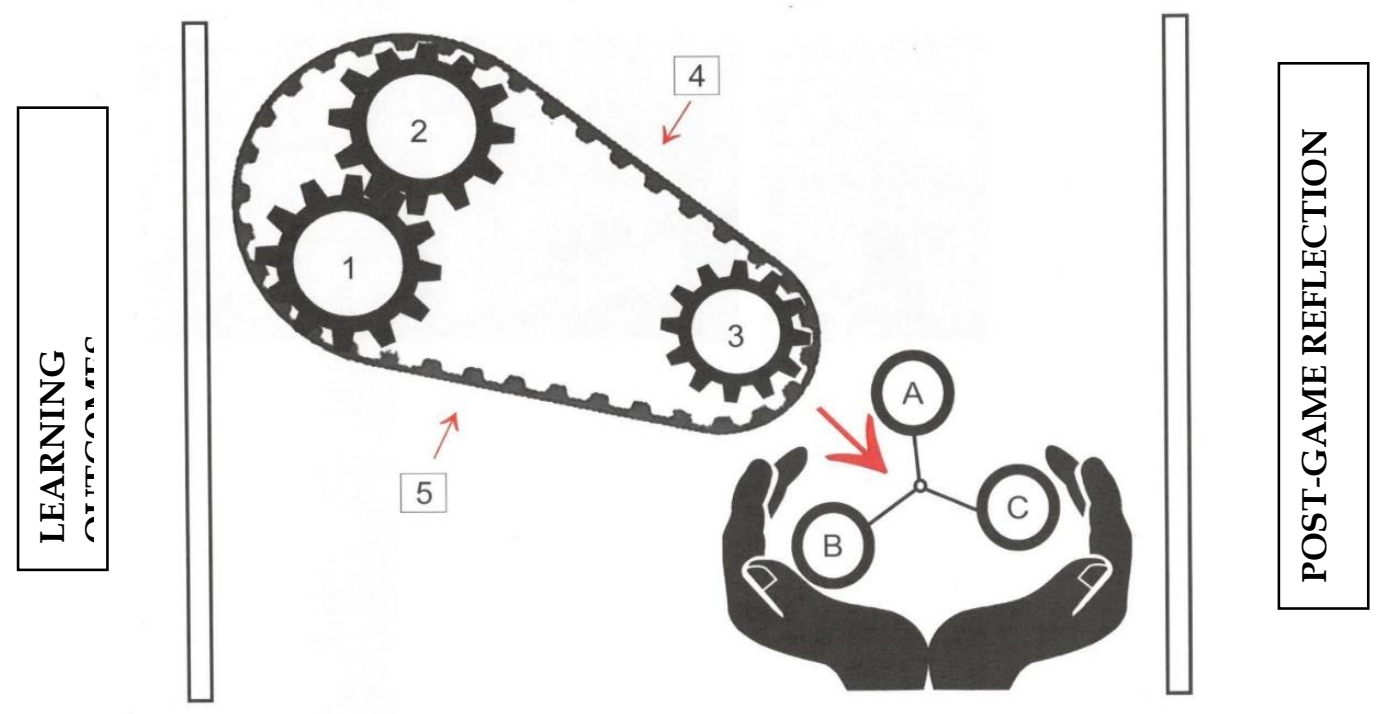

SECONDARY DRIVERS

1. INVOLVEMENT/EXPERIENCE

2. FUN/ENJOYMENT/EXCITEMENT

RESULTING IN

3. DEEPER CONCEPTUAL UNDERSTANDING
BEHAVIOURAL AND EMOTIONAL BUY-IN THROUGH ENGAGEMENT AND IMMERSION IN GAMES RESULTING IN

4. AUTONOMY; COMPETENCE: RELATEDNESS; FEEDBACK

COGNITIVE ENGAGEMENT: ENACTMENT OF KNOWLEDGE (FEEDS BACK INTO THE SYSTEM)

5. EXPERIMENTATION, STRATEGY DEVELOPMENT, REASONING, CRITICAL THINKING
THIS LEARNING PROCESS

CULMINATES IN THE ASSIMILATION OF KNOWLEDGE THROUGH
A. AWARENESS
B. REALIZATION
C. INTEGRATION/ APPLICATION

Figure 1: Game Analysis Framework (GAF) 
As depicted in Figure 1, the learning process from the gaming activity in the GAF model occurs between two pillars, namely Learning Outcomes and Post-Game Reflection, both of which remain essential components as they provide the structure within which the deliberative activity and transformational play take place. The Learning Outcomes provide the starting point where the challenges of the game are set and embedded with educational outcomes. On the other side, Post-Game Reflection represents the end of the gaming process where participants now discuss their learning experiences from the game. This allows for the development of a clearer linkage between challenges of the game and the educational outcomes paving the way for deeper conceptual understanding

Between the two pillars is the learning environment, which is represented by the Game Space within which the gaming activity takes place. The primary driver revealed by the IQA process - 'Use More Games' and the primary outcome - 'Did not Feel Like a Lesson' are catalysts of the Game Space, and it is their interaction that facilitates the disruption of the traditional educational learning space by introducing active learning into the classroom.

It is within this Game Space, that 'how' and 'why' students learn from an educational gaming activity is modelled. This takes the form of two processes: the first of which is a cogs-and-belt system that unlocks the 'safe' of deeper conceptual understanding ('how') and the second illustrates 'why' students learn from the educational gaming intervention after reaching that 'aha' moment.

Two main cogs drive the cogs-and-belt system: the first of which - 'Involvement/ Experience'-initiates the momentum of the belt-driven system by interlinking directly with the second cog - 'Fun/ Enjoyment/ Experience'. There is a reciprocal relationship between the two main cogs whereby their combined interaction adds greater traction to the learning process. This traction, in turn, sets in motion the belt that drives the third cog, namely 'deeper conceptual understanding'. Once the belt is in motion, this becomes a continuous process driven by the cogs.

The belt itself comprises two components - behavioral and emotional engagement (4) and the enactment of knowledge (5). The former, behavioral and emotional buy-in, immerses students in the gaming process by encouraging autonomy and facilitating competence and relatedness that is complemented by immediate feedback. This then leads to the second stage - the enactment of knowledge that allows for cognitive engagement, which includes experimentation, strategy development, goal formation, reasoning and critical thinking that then loop into the system.

Through students, this educational gaming intervention, are now empowered to take ownership of this deeper conceptual understanding and personalize it, as their knowledge is no longer merely academic. The game has provided the means for them to link theory to everyday occurrences in the real world. The illustration now depicts this access to awareness, realization and integration/application unlocked by deeper conceptual understanding, which is within the hands of the students and who are then enabled to create personalized meaning of the theoretical concepts. 
The model points to games providing a learning system that allows for a deeper understanding of concepts and a more meaningful application of knowledge. The question that arose was: 'How could this be possible? Part of the answer, is provided by the Cognitive Load Theory (CLT) developed by Sweller (1988). CLT provides a framework that focuses on the management of working memory as learners interact with the instructional material to achieve effective learning (de Araujo et al., 2016). If the finite working memory is exceeded, then there will a decrease in the efficacy of learning, resulting in poor retention of knowledge. Therefore, the design of instructional material should, according to CLT, manage the load of working memory to enhance the efficacy of learning. This means designing instructional material in a manner that manages the extraneous and intrinsic load that creates space within the working memory for the optimization the germane load. (de Araujo et al., 2016).

\section{Discussion}

In this section, we examine how games used in the gaming intervention reduce the extraneous load and the intrinsic load so that there is room for the expansion of the germane load, allowing for a deeper conceptual understanding of the Economics topics covered.

In this case study, three games were used to teach Microeconomics topics: Holt (1996) 'Trading in a Pit Market', Kruse et al. (2005) 'Market Forces and Price Ceilings: A Classroom Experiment' and Neral (1993) 'Widget Production in the Classroom'.

\subsection{Reducing Extraneous Load}

What follows is a step-by-step look at how games reduce/manage the extraneous load laid out as per Table 1: Some Effects Studied by Cognitive Load Theory and Why They Reduce the Extraneous Load.

\section{Goal-free effect}

The educational games focused the players on specific tasks at hand. For example, in the 'Pit Market Trading game', all the participant had to focus on was either being a buyer or seller in a market, trading to get the best price. They did not have to worry about focusing on demand and supply, definitions, diagrams or equilibrium prices. They were simply a buyer or seller aimed at making the best trade possible. By the end of successive trading rounds, participants began to notice that the successful trade prices were tending to equilibrium.

\section{Worked example effect}

An effective means of reducing the extraneous load is through the use of worked examples, which are structured in such a way that students have to follow a stepby-step process to find the solution to complex problems (Renkl, 1997). By introducing games into the Economics classroom, students actively participate in authentic, complex tasks that are scaffolded in difficulty as they progress from the one challenge to the next, culminating in a deep conceptual understanding of the problem.

The three Microeconomics games included in the Economics gaming intervention, namely: the Pit Market Trading game (Holt, 1996), Price Ceiling game (Kruse et 
al., 2005) and Widget game (Neral, 1993). These games created simulated environments in which students were economic agents working through a series of embedded tasks to reach the learning outcomes of the games. This structured learning environment allowed for students to focus their attention on the specific tasks at hand, compartmentalizing actions and thereby allowing them to know precisely what they had to do at each step of the game. In this manner, they reduced their extraneous load. For instance, in the Price Ceiling game (Kruse et al., 2005), students were allocated roles as landlords or renters. Initially, a free market reigned in the market for accommodation, based solely on the interaction between the landlords and the renters. The learning outcome at this stage was to show that markets converged to equilibrium. Then the complexity was added, namely the implementation of a price ceiling. Now, the landlords were governed by a maximum price (price ceiling) that they could charge as a rental. As a result, market conditions changed.

According to Sweller (1988; 2004), worked-out examples reduce the extraneous load by freeing up the working memory and enabling students to concentrate their attention on solving the tasks at hand. By scaffolding the tasks, less working memory is needed to be allocated to processing each task because the student only needs to solve one challenge at a time, rather than the task as a whole.

As a result, these worked-out examples as provided by the gaming intervention increase the germane cognitive load because:

"they enhance understanding of the solution procedure" (Paas \& van Gog, 2006, p. 5).

In fact, not only do the students know:

"the procedural steps for problem-solving tasks, but also understand when to deploy them and why they work" (Gott et al., 1993, p. 260).

The quotation below captures this idea that as the students followed rules of the game, the knowledge of economics was being built up and being actively learned - their minds were set free to think actively.

"Since the things that we know, you have to study it and when you have to study it, you have to grasp it. The game in order for the game to play, you have to know the rules. In order for the Economics, to know it, you have to discuss the information given to you... So then while they would be that doing in the lecture room, the knowledge it would be coming left and right asking questions because it is free, my mind is free my mind is fresh, the reason being it is a game but it is a lesson"1

\section{Completion problem effect}

The games in the intervention take conventional problems and break them down into a series of challenges that need to be completed step-by-step. As each challenge is completed, the participant moves closer to a fuller understanding of the topic being portrayed.

\footnotetext{
${ }^{1}$ Actual quote from the study.
} 
For example, the second game was based on Kruse et al. 'Market Forces and Price Ceilings: A Classroom Experiment' (2005), to show students the effect of a price ceiling on the market. The learning outcome of the game was to demonstrate how a price ceiling affects the equilibrium process.

The class was divided into groups, taking on the roles of landlords and renters in a market for student accommodation. Record sheets were distributed to the landlords as well as the renters, where the landlords' record sheet contained the costs involved with maintaining the buildings (for each period) and the renters' sheet contained a budget for rental accommodation (for each period).

At the start of the trading period, each of the landlords wrote down their rental price on a piece of paper based on their costs, which was then affixed to the wall. The renters then, in sequential order, decided whether they wanted to rent the accommodation or not, based on their budgets. Once the renter agreed to the landlord's price for a specific apartment, the accommodation was considered to be rented out, and then a line was drawn through the rental price on offer. The trading period ended once each renter had an opportunity to either rent, or decide not to rent, any of the available accommodation.

For the participants to get used to their roles (either as renters or landlords), the first four trading periods were unrestricted (i.e. the market was free of any intervention). This enabled the students to experience how the market converged towards equilibrium. When the fifth trading period began, the government intervened in the market. It imposed a price ceiling on the rentals as part of a policy to assist students with the affordability of accommodation. The landlords then had to consider the imposed price as well as their costs for the accommodation (during that period) and make a decision as to whether or not it was viable to rent their apartments. As a result, only a few apartments were available to rent at that price ceiling. On the other hand, most of the renters were competing to rent the apartments at that price because it fell within their budgets. This meant that the quantity demanded for apartments, as shown by the number of renters, exceeded the quantity of apartments supplied, causing a shortage (excess demand) for apartments in the market.

Therefore, the overarching learning outcome of understanding the effect of a price ceiling was broken down into two parts:

- $\quad$ Part 1: where the free trading between landlords and renters in various scenarios tends to equilibrium; and

- $\quad$ Part 2: where the government introduces a price ceiling, and a shortage in the market is experienced.

\section{Split-attention effect}

In the gaming environment, student attention is focused on what is needed to complete the task within the parameters of the game. In the Neral's (1993) Widget production game, the focus was on producing the widgets in each round of production. The attention of the student was not split amongst multiple sources of information such as diagrams, tables and definitions that would be used to 
describe the theory being portrayed in a traditional lecture, but instead focused on the task.

This focus on the widget production only enabled them to follow the data they generated and to draw conclusions on their lived experience of the game, without being side-tracked by mathematical formulas and definitions that usually accompany the traditional lecture on the Law of Diminishing Returns. This resulted in reducing the extraneous load and making space for the germane load - where students can to reflect and develop new schemata based on their learning experience in the gaming environment.

\section{Modality effect}

As games harness the learners' audio and visual senses simultaneously, their capacity to process information is enhanced. "'Physically integrating multiple sources of information facilitates learning by reducing working memory load"' (Mousavi et al., 1995, p.320). Therefore, by reducing the learners' dependence on one sensory channel (which could easily get saturated because it is finite) and spreading the load across two or more channels, their working memory capacity is expanded. This is referred to as 'off-loading'. Thus, when a learner receives verbal and visual instructions simultaneously, it will generally cause them to learn more efficiently than if the same instructions were received in only one format (Mayer \& Moreno, 2003). Games tend to create environments that encourage the use of multiple sensory channels, ranging from visual to auditory to tactile, resulting in a vivid learning experience.

"We tend to capture them easier than words. I think it is because of the colours, the pictures, the fun for everyone. You see the faces, who did what, who was part of this group which makes you remember when you are studying that ... Ja, so I think when you capture something it is easier for your brain to store and keeping the information in than when you are reading from a textbook"2

\section{Redundancy effect}

The game space becomes the single source of information that the students required to complete the tasks at hand, whether trading with each other in the Pit Market trading game as a buyer or seller; being a landlord or renter Price Ceiling game; or that of a firm trying to produce widgets. The rules of the game govern the participants' behavior as they haggle, trade and produce goods, thereby reducing the extraneous load. Moreover, participants do not need to search for information beyond the structure and rules of the game.

\subsection{Reducing the intrinsic load}

In this section, we examine how games used in the gaming intervention reduce the intrinsic load and make room for the expansion of the germane load that allows for a deeper conceptual understanding of the Economics topics covered.

\section{Level of Expertise}

The games played in this intervention were not done in isolation but embedded with the curriculum so that when it came to playing the game, learners had some

${ }^{2}$ Actual quote from study. 
understanding of the concepts being taught. For example, the second game was based on Kruse et al. 'Market Forces and Price Ceilings: A Classroom Experiment' (2005), to show students the effect of a price ceiling on the market. The learning outcome of the game was to demonstrate how a price ceiling affects the equilibrium process.

The students already had prerequisite knowledge about equilibrium in a market, which means that all they had to do was add another layer of understanding on concepts they already knew, thereby lowering the intrinsic load.

Also, the games were not played as single participants but rather in groups, with each person getting a chance to participate. This means that the student had access to extra levels of expertise offered by other students within the group. Furthermore, the group had access to the lecturer if they needed help as the lecturer was available and was no longer caught up in teaching but was instead a game host, having time to answer queries. This may have also helped to reduce the intrinsic load of the game.

\section{Reducing the difficulty}

The games, through rounds of playing, slowly built up towards the complex final outcome. This sounds very similar to the Work Example and Completion problem effect under approaches to reduce the extraneous load. They are very similar in that they break down a complex problem into a series of events that, when followed, result in the 'Aha' moment - where all the steps are linked together to gain a deeper conceptual understanding of the topic.

For example, in Holt (1996) 'Trading in a Pit Market', the students trade as a buyer or seller. They see, through successive rounds of trading between buyers and sellers, that a market equilibrium price is reached. This conclusion is then also reinforced by post-game reflections which add to the deeper conceptual understanding.

\section{Conclusion}

Games can create complex learning environments that are able to manage the cognitive load in a way that facilitates an optimal usage of working memory, resulting in effective learning. Managing the extraneous and intrinsic load is paramount if the germane load is to be optimized and expanded. The germane load is where schemata are created and retained in long-term memory, thereby ensuring that learning lasts beyond the classroom.

CLT theory provides insight into 'how' and 'why' games can create a deeper conceptual understanding, giving it legitimacy as a medium of instruction. In more general terms CLT, prompts instructional designers focus more sharply on:

"(1) Investigating intrinsic cognitive load helps in understanding the subjective complexity placed on the learners, (2) investigating extraneous cognitive load helps in understanding the impact of differences in design, and (3) investigating germane cognitive load helps to understand the effort learners invested in learning." (Klepsch \& Seufert, 2020, p. 47) 
Such a process of reflecting on the educational design, whether for games, experiential learning or conventional lectures, will lead to learning environments that improve the quality of learning. Perhaps it should become the norm when designing learning environment.

In terms of future research in relation to games and CLT, it is further proposed that we look at how games not only are able to reduce intrinsic and extraneous loads, but also how they ensure that the additional space created in the working memory is allocated to the development of new knowledge - increasing the germane load.

In addition, research comparing the cognitive loads between games and other mediums of instruction may yield interesting results that would add to the body of existing knowledge. In terms CLT, further research needs to be undertaken in looking at how the intrinsic load can also be managed to create space for a more optimal use of the germane load in the working memory.

\section{References}

Ball, S., Eckel, C., \& Rojas, C. (2006). Technology improves learning in large principles of Economics classes: Using our WITS. American Economic Review, 96(2), 442-446. Retrieved from https:// ssrn.com/abstract $=1843411$

Davis, J. S. (2011). Games and Students: Perceptions and Evidence from a DUT Case Study. International Journal of Business and Economic Research (IBER), 10(10). doi:10.19030/iber.v10i10.5975

de Araujo, G. G. T., de Jorge, B., Franci, D., Martins, S. T., Vellutini, S. M. S., Schweller, M., \& de Carvalho-Filho, M. A. (2016). Cognitive load and self-determination theories applied to e-learning: Impact on students' participation and academic performance. PLoS ONE, 11(3), Article e0152462. doi:10.1371/journal.pone.0152462

Emerson, T. L., \& Taylor, B. A. (2004). Comparing student achievement across experimental and lecture-oriented sections of a principles of micro-economics course. Southern Economic Journal, 70(3), 672-93.

Gott, S. P., Parker Hall, E., Pokorny, R. A., Dibble, E., \& Glaser, R. (1993). A naturalistic study of transfer: Adaptive expertise in technical domains. In D. K. Detterman and R. J. Sternberg (Eds.) Transfer on trial: Intelligence, cognition, and instruction, 258-288. Norwood, NJ: Ablex.

Gremmen, H., \& van den Brekel, G. (2013). Do Classroom Experiments Increase Student Motivation? A Pilot Study. European Scientific Journal, ESJ, 9(19). doi:10.19044/esj.2013.v9n19p\%p

Hays, R. (2005). The Effectiveness of Instructional Games: A Literature Review and Discussion. Orlando. FL.: Technical Report, Naval Air Warfare Center Training Systems Division.

Hawlitschek, A., \& Joeckel, S. (2017) Increasing the effectiveness of digital educational games: The effects of a learning instruction on students' learning, motivation and cognitive load. Computers in Human Behavior, 72, 79-86. doi:10.1016/j.chb.2017.01.040

Holt, C. A. (1996). Classroom Games: Trading in the Pit. Journal of Economic Perspectives, 10(1), 193-203. doi:10.1257/jep.10.1.193

Klepsch, M., \& Seufert, T. (2020). Understanding instructional design effects by differentiated measurement of intrinsic, extraneous, and germane cognitive load. Instructional Science, 48, 45-77. doi:10.1007/s11251-020-09502-9 
Kruse, J. B., Ozdemir, O., \& Thompson, M. A. (2005). Market Forces and Price Ceilings: A Classroom Experiment. International Review of Economics Education, 4(2), 73-86. doi:10.1016/S1477-3880(15)30130-4

Mayer, R. E., \& Moreno, R. (2003). Nine Ways to Reduce Cognitive Load in Multimedia Learning. Educational Psychologist, 38(1), 43-52. doi:10.1207/S15326985EP3801

Mousavi, S. Y., Low, R., \& Sweller, J. (1995). Reducing Cognitive Load by Mixing Auditory and Visual Presentation Modes. Journal of Educational Psychology, 87(2), 319-334. doi:10.1037/0022-0663.87.2.319

Neral, J. (1993). Widget Production in the Classroom. Classroom Expernomics, 2(1).

Nkonyane, V. A., \& Van Wyk, M. M. (2015). Post Graduate Certificate of Education Student Teachers' Views of Economics Games as an Interactive Classroom Technique. International Journal of Education Science, 8(2), 427-434. doi:10.1080/09751122.2015.11890264

Northcutt, N., \& McCoy, D. (2004). Interactive Qualitative Analysis: A Systems Method for Qualitative Research. Sage Publications.

Ouellette, M., Breeding, L., \& Clark, C. (2019) Using applied cognitive load theory and difficulty analysis for educational game design for understanding and transference of literacy skills in adults. FDG '19: Proceedings of the 14th International Conference on the Foundations of Digital Games (pp. 1-11). San Luis Obispo, California. doi:10.1145/3337722.3337725

Paas, F., \& van Gog, T. (2006). Optimising worked example instruction: Different ways to increase germane cognitive load. Learning and Instruction, 16, 87-91. doi:10.1016/j.learninstruc.2006.02.004

Randel, J. M., Morris, B. A., Wetzel, C. D., \& Whitehill, B. V. (1992). The Effectiveness of Games for Educational Purposes: A Review of Recent Research. Simulation Gaming, 23(3), 261-276. doi:10.1177/1046878192233001

Renkl, A. (1997). Learning from Worked-Out Examples: A Study on Individual Differences. Cognitive Science, 21(1), 1-29. doi:10.1016/S0364-0213(99)80017-2

Sitzmann, T. (2011). A Meta-Analytic Examination of the Instructional Effectiveness of Computer-based Simulation Games. Personnel Psychology, 64, 489-528. doi:10.1111/j.1744-6570.2011.01190.x

Sweller, J. (1988). Cognitive load during problem solving: Effects on learning. Cognitive Science, 12, 257-285. doi:10.1207/s15516709cog1202_4

Sweller, J. (2004). Instructional Design Consequences of an Analogy between Evolution by Natural Selection and Human Cognitive Architecture. Instructional Science, 32, 931. doi:10.1023/B:TRUC.0000021808.72598.4d

Sweller, J., van Merriënboer, J. J. G., \& Paas, F. (2019). Cognitive Architecture and Instructional Design: 20 Years Late. Educational Psychology Review, 31, 261-292. doi:10.1007/s10648-019-09465-5

Tsigaris, P. (2008). Is There A Double Dividend From Classroom Experimental Games? American Journal of Business Education (AJBE), 1(1), 23-36. doi:10.19030/ajbe.v1i1.4634

Young J. Q., Van Merrienboer, J., Durning, S., \& Ten Cate, O. (2014). Cognitive Load Theory: implications for medical education: AMEE Guide No. 86. Medical Teacher, 36(5), 371-384. doi:10.3109/0142159X.2014.889290

van Merrienboer, J. J. G., \& Sweller, J. (2005). Cognitive Load Theory and Complex Learning: Recent Developments and Future Directions. Educational Psychology Review, 17(2), 147-177. doi:10.1007/s10648-005-3951-0

Vlachopoulos, D., \& Makri, A. (2017) The effect of games and simulations on higher education: a systematic literature review. International Journal of Educational Technology in Higher Education, 14, 22. doi:10.1186/s41239-017-0062-1 
Vogel, J. J., Vogel, D. S., Cannon-Bowers, J., Bowers, C. A., Muse, K., \& Wright, M. (2006). Computer Gaming and Interactive Simulations for Learning: A Meta-Analysis. Journal of Educational Computing Research, 34(3), 229-243. doi:10.2190/FLHVK4WA-WPVQ-HOYM

Wouters, P., van Oostendorp, H., van Nimwegen, C., \& van der Spek, E. (2013). Metaanalysis of the Cognitive and Motivational Effects of Serious Games. Journal of Educational Psychology, 60(1), 412-425. doi:10.1037/a0031311

Young, J. Q., Van Merrienboer, J., Durning, S., \& Ten Cate, O. (2014). Cognitive Load Theory: implications for medical education: AMEE Guide No. 86. Medical Teacher, 36(5), 371-384. doi:10.3109/0142159X.2014.889290 